Collection SFN 11 (2010) 137-151

(C) Owned by the authors, published by EDP Sciences, 2010

DOI: $10.1051 / \mathrm{sfn} / 201011009$

\title{
Interfaces de polymères en couche minces : la réflectivité des neutrons
}

\author{
M. Sferrazza et W. Béziel
}

Département de Physique, Faculté des Sciences, Université Libre de Bruxelles, Boulevard du Triomphe CP223, 1050 Bruxelles, Belgique

\begin{abstract}
Résumé. La réflectivité des neutrons est utilisée pour l'étude de la structure de l'interface entre couches minces de polymères afin de sonder les fluctuations thermiques et les effets de confinement dans ces systèmes. Des exemples d'étude sont illustrés dans ce chapitre: l'interface entre deux films minces de polymères est étudiée en fonction du degré d'immiscibilité, de l'épaisseur des films et du temps, i.e de la cinétique de formation de l'interface.
\end{abstract}

\section{INTRODUCTION}

Les propriétés des surfaces et interfaces de polymères sont cruciales dans beaucoup d'applications, telles que l'adhésion, les traitements contre la corrosion mais aussi le développement de composants en microélectronique. La compréhension des processus physiques, au niveau nanométrique, à ces interfaces de polymères est donc importante pour les applications industrielles. L'étude de la nature de l'interface entre deux liquides en coexistence a récemment connu un essor considérable, en particulier celui lié aux effets de confinement et d'une dimensionnalité réduite de la phase liquide. Ce sujet est lié à plusieurs problèmes technologiques, comme par exemple le comportement de phase d'un mélange fluidique dans des milieux poreux, ou encore la formation de multicouches dans des procédures industrielles. De plus, il peut être avantageux d'utiliser les polymères comme modèles pour les études générales du comportement de phases de films minces confinés [1] car d'une part la longueur d'échelle intrinsèque, le rayon de giration de la chaine, simplifie les expériences et l'interprétation théorique permettant la séparation entre les différentes longueurs du système (atomique et moléculaire), et d'autre part, les polymères sont caractérisés par une dynamique lente. Ces avantages permettent de sonder la dynamique du système s'approchant de l'équilibre et autorise également l'étude des états métastables. Au point de vue de la préparation des couches minces de polymères, des techniques simples, comme le "spincoating", peuvent être utilisées pour la préparation des films de polymères avec un contrôle d'épaisseur allant de quelques nanomètres à quelques centaines de nanomètres. La préparation de bicouche (ou multicouche) de polymère ayant une interface nette de l'ordre de l'angstroem ( $)$ et hors de son état d'équilibre est donc relativement simple. Pour l'étude de cette interface la technique de la réflectivité des neutrons est idéale [2] [3], car sa résolution est de l'ordre de l'Å et elle offre la possibilité d'utiliser la technique de deuteration, des bicouches avec un polymère deuteré et l'autre hydrogéné, ce qui permet l'étude en détail du profil de l'interface.

L'interface entre deux films de polymères n'est pas nette au niveau atomique: il y a une compétition entre l'enthalpie défavorable au mélange au niveau de l'interface diffuse et le gain d'entropie des chaînes. La théorie des champs auto-consistants prédit l'épaisseur de l'interface et la tension interfaciale pour un couple de polymères immiscibles. Le profil de la fraction volumique d'un des composants suit

This is an Open Access article distributed under the terms of the Creative Commons Attribution-Noncommercial License 3.0, which permits unrestricted use, distribution, and reproduction in any noncommercial medium, provided the original work is properly cited. 
la forme d'une tangente hyperbolique et l'épaisseur de l'interface varie comme $\chi^{-1 / 2}$ pour des petits $\chi$ ( $\chi$ est le paramètre d'interaction de Flory-Huggins), tandis que la tension interfaciale est proportionnelle à $\chi^{1 / 2}[4]$.

La valeur expérimentale de l'épaisseur de l'interface pour des systèmes incompatibles est plus large que la valeur prédite par la théorie des champs auto-consistants (Helfand and Tagami) [4]. Pour comprendre cette différence, les fluctuations thermiques aux interfaces doivent être considérées [5, 6]. Dans ce chapitre, nous montrerons que la technique de la réflectivité de neutrons est idéale pour étudier ces fluctuations thermiques, les effets de confinement et la dynamique de formation de l'interface.

\section{L'INTERFACE ENTRE DEUX POLYMÈRES}

Pour décrire le profil de l'interface entre deux polymères pour différents cas de miscibilités, deux théories différentes, la théorie des champs auto-consistants [2, 4, 7], et la « Square Gradient» théorie [8] [9] peuvent être utilisées. Dans la théorie des champs auto-consistants, l'équation de diffusion d'une chaine de polymère est donnée par l'équation 1 . Le premier terme de l'équation représente une marche aléatoire de la chaîne de polymères à l'interface dans un espace libre auquel on ajoute un terme représentant l'effet d'un potentiel spatial. On définit donc une fonction de distribution q $\left(\mathrm{r}, \mathrm{r}^{\prime}, \mathrm{t}\right)$ qui représente la probabilité qu'une chaîne de $t$ segments commence à une position $r$ et finisse à une position $\mathrm{r}^{\prime}[4]$ :

$$
\frac{\partial q\left(r, r^{\prime}, t\right)}{\partial \mathrm{t}}=\frac{a^{2}}{6} \nabla^{2} q\left(r, r^{\prime}, t\right)-\frac{U(r)}{k T} q\left(r, r^{\prime}, t\right)
$$

où $a$ est la longueur caractéristique d'un segment de polymère, $k_{B}$ la constante de Boltzmann, T la température. U(r) est le potentiel de variation spatiale définit par :

$$
U(r)=\chi(1-\varphi(r))+w(r)
$$

où $\varphi(\mathrm{r})$ représente la fraction volumique d'une espèce en fonction de la position (dans l'équation 2 le premier terme représente l'interaction entre les différents monomères tandis que w(r) est lié à l'incompressibilité du système [2,4]). Si on étudie la diffusion selon l'axe $\mathrm{z}$, la fraction volumique va s'exprimer par :

$$
\varphi_{A}(z)=\frac{1}{N} \int_{0}^{N} d t q_{A}(z, N-t) q_{A}(z, t) .
$$

Il faut appliquer ces équations aux deux types de polymères en les considérants dans une géométrie d'interface planaire. On obtient ainsi un système d'équations différentielles. Pour les résoudre on définit les $\varphi_{A}(\mathrm{z})$ et $\varphi_{B}(\mathrm{z})$ des deux espèces $\mathrm{A}$ et $\mathrm{B}$ arbitrairement, ensuite on calcule les potentiels de variation spatiale, et enfin on résout les équations donnant les fonctions de distributions. Ainsi on a une description pour $\varphi_{A}(\mathrm{z})$ et $\varphi_{B}(\mathrm{z})$. Et on recommence ce processus un certains nombre de fois [10].

Dans l'approximation de deux polymères immiscibles ayant des masses moléculaires tendant vers l'infini, une solution analytique exacte existe [2]. La fraction volumique de l'espèce A, selon l'axe z, est décrite par:

$$
\varphi_{\mathrm{A}}(z)=\frac{1}{2}\left[1+\tanh \left(\frac{z}{\mathrm{w}_{I}}\right)\right]
$$

où $\mathrm{w}_{I}$ représente l'épaisseur de l'interface intrinsèque, et est défini par :

$$
w_{\mathrm{I}}=\frac{a}{\sqrt{6 \chi}}
$$


et la tension interfaciale, $\gamma$, entre deux phases, est donnée par :

$$
\gamma=k_{B} \mathrm{~T} \rho a \sqrt{\frac{\chi}{6}}
$$

où $\rho$ correspond à la densité des polymères. Dans cette théorie de l'interface entre deux polymères, la longueur des chaînes a été supposée infinie $(\mathrm{N} \sim \infty)$. Il faut aussi considérer l'effet de la longueur finie de chaine et les équations 5 et 6 deviennent [11]:

$$
\begin{gathered}
w_{I}=\frac{\mathrm{a}}{\sqrt{6 \mathrm{v}}}\left[\frac{3}{4}\left(1-\frac{2}{\chi N}\right)+\frac{1}{4}\left(1-\frac{2}{\chi N}\right)^{2}\right]^{-1 / 2} \\
\gamma=k_{B} \mathrm{~T} \rho a \sqrt{\frac{\chi}{6}}\left(1-\frac{1.8}{\chi N}-\frac{0.4}{(\chi N)^{2}}\right)^{3 / 2} .
\end{gathered}
$$

Ces ajustements ont été proposés par Tang and Freed [11]. Le modèle présenté décrit l'interface dans le cas de la «Strong-Segregation-Limit» (SSL), où l'interface qui sépare les deux polymères est fortement immiscible. Dans le cas où les polymères sont légèrement immiscibles, cette description n'est plus valide. La théorie proposée par Chan and Hilliard [12] introduit la présence d'un gradient dans la composition de l'interface. L'énergie libre va être donnée par la somme de deux termes :

$$
\mathrm{F}_{\mathrm{SQ}}=k_{B} T \int\left\lfloor\mathrm{F}_{F H}(\varphi)+K(\varphi)(\nabla \varphi)^{2}\right\rfloor \mathrm{d} r .
$$

Le premier correspond à l'énergie libre de Flory-Huggins et le second terme est proportionnel au carré du gradient de composition. A partir de cette relation les expressions de l'interface intrinsèque et de la tension de surface deviennent :

$$
\begin{aligned}
& w_{\text {crit }}=\frac{a \sqrt{N}}{3}\left(\frac{\chi}{\chi_{\text {crit }}}-1\right)^{-1 / 2} \\
& \gamma_{\text {crit }}=\frac{9 k_{B} T}{a^{2} \sqrt{N}}\left(1-\frac{\chi_{\text {crit }}}{\chi}\right)^{3 / 2}
\end{aligned}
$$

où $\chi_{\text {crit }}$ est la valeur du paramètre de Flory-Huggins $\chi$ au point critique. Ce modèle décrit l'interface dans le cas de la «Weak-Segregation-Limit» (WSL), où l'interface qui sépare deux polymères est légèrement immiscible. Maintenant que l'épaisseur de l'interface intrinsèque a été décrite, il ne reste plus qu'à introduire les ondes capillaires à l'interface $[13,14]$. Elles représentent les fluctuations thermiques locales de l'interface. On décrit la surface $\zeta(\mathrm{x}, \mathrm{y})$ comme étant une somme d'ondes :

$$
\varsigma(x, y)=\sum_{k} A(q) e^{i \vec{q} \cdot \vec{s}}
$$

$\vec{S}$ correspond à un vecteur dans le plan $(\mathrm{x}, \mathrm{y}), \overrightarrow{\mathrm{q}}$ à un vecteur d'onde et $\mathrm{A}(\mathrm{q})$ à l'amplitude du même vecteur d'onde. On peut ensuite calculer le carré de la moyenne de l'amplitude des ondes capillaires $\sigma_{\zeta}^{2}[13]:$

$$
\sigma_{\varsigma}^{2}=\left\langle\varsigma^{2}-\langle\varsigma\rangle^{2}\right\rangle=\int \frac{k_{B} T}{2 \pi \gamma} \frac{d q}{q} .
$$

Cependant l'intégrale diverge à l'interface, donc pour les limites il faut reprendre les limites physiques. On obtient finalement :

$$
\sigma_{\varsigma}^{2}=\frac{k_{B} T}{2 \gamma \pi} \ln \frac{q_{\max }}{q_{\min }} .
$$


où $\mathrm{q}_{\max }$ et $\mathrm{q}_{\min }$ correspondent aux bornes inférieure et supérieure de l'onde capillaire, liées respectivement à l'épaisseur de l'interface et généralement à la longueur de cohérence des neutrons. On peut donc enfin décrire l'épaisseur de l'interface totale par :

$$
2 w=\left(4 w_{I}^{2}+2 \pi \sigma_{\varsigma}^{2}\right)^{1 / 2} .
$$

\section{EFFETS DE CONFINEMENTS SUR LES FLUCTUATIONS INTERFACIALES}

Dans la section précédente, il a été abordé que l'épaisseur interfaciale entre deux polymères est influencée par des potentiels qui affectent le comportement des ondes capillaires. Pour les films minces de polymères deux comportements différents sont expérimentalement observés. Ils relient la taille des systèmes à l'épaisseur de l'interface. Par exemple, des expériences sur des polymères fortement immiscibles -polystyrène/poly(méthylmethacrylate) (PS/PMMA)- ont montré que les forces dispersives à longue portée, dans des films très minces, jouent le rôle de «cut-off » pour les petites longueurs d'ondes et qu'une dépendance logarithmique de l'épaisseur de l'interface est obtenue avec la taille du film [15-17].

\subsection{Les forces dispersives à longue portée}

Les forces de Van der Waals - interactions dipôles-dipôles entre des atomes ou des molécules - sont effectives à longue portées et toujours présentes même dans des systèmes neutres [18]. Elles jouent également un rôle important dans beaucoup de phénomènes physiques comme le mouillage, l'adhésion ou encore l'absorption. Dans le cas d'une interface entre deux polymères, les forces dispersives deviennent non négligeables si l'un des deux ou les deux milieux sont plutôt fins. Quand un film mince est interposé entre deux milieux semi-infinis, comme par exemple un polymère entre un substrat et un autre milieu, les interactions de Van der Waals entre les deux milieux à travers le film doivent être considérées. La force dispersive est impliquée dans la borne inférieure des petits vecteurs d'ondes ; elle supprime les ondes capillaires avec des longueurs d'ondes plus grandes que la longueur d'onde capillaire caractéristique $\mathrm{a}_{\mathrm{disp}}$ :

$$
a_{\text {disp }}=\sqrt{\frac{4 \pi \gamma d^{4}}{|A|}}
$$

où $\mathrm{A}$ est la constante de Hamaker et $\mathrm{d}$ l'épaisseur de la couche. La valeur de $\mathrm{q}_{\text {min }}$ dans l'équation 14 est égale à $2 \pi / \mathrm{a}_{\text {disp }}$. Il en résulte que la contribution des ondes capillaires dans l'épaisseur interfaciale est réduite à une dépendance logarithmique de l'interface en fonction de l'épaisseur du film, comme il a déjà été observé lors d'expériences de réflectivité de neutrons par Sferrazza et al. [15].

\subsection{Les forces à courte portée}

Pour les couples de polymères quasiment miscibles, et donc avec des larges épaisseurs de l'interface, d'autres effets reliés à la taille finie du système doivent être pris en compte. Quand la largeur de l'interface est une fraction importante de l'épaisseur du film, il y a des interactions à courte portée entre l'interface et les parois du film. Une molécule de polymère à l'interface est donc soumise dans ce cas à un potentiel effectif qui prend la forme de [19-21] :

$$
V(d) \propto e^{-k d}
$$

où $\mathrm{d}$ est la distance de l'interface par rapport à la surface. La surface aura pour effet de tronquer le profil de l'interface, en introduisant une longueur de corrélation $\xi$ sur les fluctuations de l'interface dans la direction parallèle au plan de l'interface, laquelle croit de manière exponentielle avec l'épaisseur du film 
d [22-24]:

$$
\xi \approx \xi_{B} e^{k d / 2}
$$

Dans l'équation 18, $\xi_{B}$ représente la longueur de corrélation en «volume» et $\mathrm{k}^{-1}$ est la diminution dela longueur transverse ( $\mathrm{k}$ est de l'ordre de $\xi_{B}$ pour les couples de polymères faiblement immiscibles). La forte dépendance de $\xi$ en fonction de l'épaisseur du film peut être comprise en considérant que les effets de "troncatures" du profil de l'interface liés à la présence des parois sont faibles dans des films épais, où les limites des films sont plus distantes de la position moyenne de l'interface. Pour des films plus fins, au contraire, l'effet devient plus important et la longueur de corrélation est plus petite. Il en résulte que le vecteur d'onde minimum $\mathrm{q}_{\text {min }}$ est donné par $2 \pi / \xi$, et en négligeant les forces à longue portée, la dispersion moyenne au carré $\sigma_{\zeta}^{2}$ des ondes capillaires contient un terme qui est proportionnel à l'épaisseur d'un film d, comme il a été montré expérimentalement par Kerle et al. [25].

En se focalisant sur un système où on peut changer de manière systématique les conditions de miscibilité, et par conséquent l'épaisseur de l'interface, les deux effets peuvent être étudiés: les forces dispersives à longue portée prédisent une dépendance logarithmique de la largeur de l'interface en fonction de l'épaisseur du film tandis que les interactions à courte portée impliquent une dépendance en fonction de la racine carrée de l'épaisseur.

\section{CINÉTIQUE DE FORMATION DE L'INTERFACE}

Les chaînes de polymère ont une dynamique lente, et il est donc possible de distinguer la formation de l'interface intrinsèque, laquelle est reliée directement à la mobilité des chaines, dans la croissance des ondes capillaires, celles-ci se font à des temps longs. Pour rappel, l'épaisseur de l'interface est composée de deux parties : une interface intrinsèque $\mathrm{w}_{I}$, et le carré de la moyenne de l'amplitude des oscillations causées par la fluctuation des ondes capillaires. Dans l'équation 14, q $\mathrm{min}_{\text {nin }}$ et $\mathrm{q}_{\max }$ sont liés respectivement au maximum et au minimum des longueurs d'onde des fluctuations. L'interface intrinsèque exprimée par la théorie SCF est une bonne approximation pour $\lambda_{\min }$. En ce qui concerne l'expression de $\lambda_{\max }$, généralement pour une interface à l'équilibre, la valeur maximale limitée par la longueur de cohérence des neutrons est de quelques micromètres. Cependant dans le cas d'une interface qui n'est pas dans son état d'équilibre, il est clair que les petites longueurs d'ondes capillaires seront dans leur état d'équilibre plus rapidement que les grandes longueurs d'ondes. Ainsi la longueur d'onde maximale dépendra du temps. Dans une étude précédente, Sferrazza et al. ont observé que le système PS/PMMA admet une dépendance logarithmique de l'interface en fonction du temps [17]. La dynamique des longueurs d'onde dans des interfaces liquide/liquide a été fortement étudiée dans le contexte de la diffusion de la lumière. Dans la limite des grandes viscosités et des faibles tensions de surface, il existe une expression simple pour le temps de relaxation $\tau$ d'une onde capillaire de vecteur d'onde $\mathrm{q}$, à une interface semi-infinie: $\tau \propto \eta /(\gamma \mathrm{q})$, où $\eta$ est la moyenne des viscosités entre les deux fluides. A partir de cette relation, pour un temps $\mathrm{t}$, toutes les ondes capillaires seront à l'équilibre pour une longueur d'onde inférieure à l(t) :

$$
l(t) \propto \frac{\gamma}{\eta} t
$$

A ce stade, l'augmentation en fonction du temps de l'épaisseur de l'interface peut donc être prédite :

$$
4 w^{2}=4 w_{I}^{2}+2 \pi\left[\frac{k_{B} T}{2 \gamma \pi} \ln \frac{(\gamma / \eta) t}{\pi w_{I}}\right] .
$$

\section{CHOIX DU SYSTÈME DE POLYMÈRES}

On s'intéresse à l'étude de la structure et formation en fonction du temps de l'interface entre deux polymères en variant le paramètre d'interaction entre les deux polymères et l'épaisseur des couches. 
Un système modèle pour ces études est le copolymère polyoléfine. Ce copolymère est un mélange des monomères d'éthylène et de butène. Il est obtenue à partir du polybutadiène, $\left(\mathrm{C}_{4} \mathrm{H}_{6}\right)_{N}$, et il peut avoir deux configurations différentes: 1-4 ou 1-2. L'utilisation des deux configurations différentes de butadiène, donne le contrôle sur le type de monomères (éthylène ou butène) polymérisé, et au final le ratio entre les deux monomères. La proportion du mélange des monomères est donnée par le paramètre $\mathrm{x}$ dans la formule chimique $\left(\mathrm{C}_{4} \mathrm{H}_{8}\right)_{1-x}\left(\mathrm{C}_{2} \mathrm{H}_{3}\left(\mathrm{C}_{2} \mathrm{H}_{5}\right)\right)_{x}$. On peut donc varier la miscibilité du système en changeant le ratio $\mathrm{x}$ dans la composition du polymère. Le choix de ce copolymère vient donc du fait qu'on peut facilement sélectionner la miscibilité des deux couches, grâce au paramètre d'interaction $\chi$ [25-27]. La $\mathrm{T}_{g}$ (température de transition vitreuse du polymère) est d'environ $-61{ }^{\circ} \mathrm{C}$.

Après la préparation, les échantillons sont donc conservés à une température de $-80^{\circ} \mathrm{C}$. Les échantillons ont été préparés par «spin-coating»: la couche deutérée est déposée directement sur le substrat de silicium, et la couche hydrogénée est «spin-coatée» sur une plaque de verre et ensuite déposée par «floating» sur la couche deutérée. Les échantillons ont subi différents temps de chauffage à $83^{\circ} \mathrm{C}$ afin d'observer les différentes étapes de la formation de l'interface. La substitution isotopique hydrogène-deutérium (H-D) peut affecter l'intéraction thermodynamique. Il a déjà été observé dans des études précédentes que le paramètre du système $\chi$ résultant décroîtra quand le mélange avec le copolymère de plus faible ratio est deutéré, alors qu'il sera plus élevé dans le cas contraire. L'erreur sur la détermination de la valeur de $\chi$ pour les ratios de combinaisons de copolymères utilisés expérimentalement est estimée à moins de $5 \%$.

\section{RÉFLECTIVITÉ DES NEUTRONS}

L'interface entre deux polymères immiscibles est typiquement plus petite que 400/500 Å. L'utilisation d'une technique pour mesurer la largeur interfaciale doit donc prendre en considération cet ordre de grandeur. De plus, un contraste adapté doit être disponible entre les deux milieux [28, 29].

La réflectivité des neutrons représente un outil idéal pour sonder les interfaces polymère/polymère. Cette technique fournit une excellente résolution spatiale plus basse que $1 \mathrm{~nm}$, due aux petites longueurs d'ondes disponibles avec les neutrons thermiques. Elle donne également la possibilité d'examiner les interfaces à l'intérieur du système peu facilement accessible, grâce à la grande capacité pénétrante des neutrons, lesquels permettent à un faisceau de neutrons incident de se propager dans un milieu solide jusqu'à atteindre une interface. Cela représente un excellent avantage pour l'étude des interfaces solideliquide [30]. De plus, le faisceau de neutrons est très peu destructif, ce qui permet plusieurs mesures d'un même échantillon. La réussite de l'application de la réflectométrie des neutrons sur des polymères repose aussi sur le fait que la densité de la longueur de diffusion d'un matériau peut être modulée en réalisant une substitution isotopique entre l'hydrogène et le deutérium. Ceci permet de générer un large contraste entre les différents milieux de polymères et, donc, d'être plus sensible à l'interface. Les expériences de réflectivité examinent la variation moyenne de l'indice de réfraction perpendiculaire aux interfaces. Donc, pour les analyses de réflexion spéculaire des neutrons sur la structure d'un film mince, le profil de la densité de la longueur de diffusion unidimensionnelle et perpendiculaire à la surface peut être obtenu. Celui-ci peut être directement relié au profil chimique.

La réflectivité est donnée par $R=|r|^{2}$, où $\mathrm{r}$ est l'amplitude de la réflectance obtenue en résolvant l'équation de Schrödinger unidimensionnelle, dans la direction perpendiculaire à l'échantillon. Si nous avons une propagation des neutrons du vide dans un matériau uniforme et de densité de longueur de diffusion $\mathrm{b} / \mathrm{V}$, la composante perpendiculaire du vecteur d'onde du matériau $k_{i}$ est exprimée par :

$$
k_{i}=\sqrt{k^{2}-4 \pi \frac{b}{V}} .
$$

Dans le cas où $4 \pi b / V>k^{2}$, k est imaginaire et les neutrons se propagent dans le matériau uniquement comme une onde évanescente donnant un flux de réflexion externe totale (réflectivité égal à l'unité). Dans le cas où $4 \pi b / V<k^{2}$ et d'une interface net, la réflectivité est donnée par les expressions de 
Fresnel :

$$
R=|r|^{2}=\left|\frac{k-k_{i}}{k+k_{i}}\right|^{2}
$$

Pour une haute valeur de $\mathrm{k}$, l'expression limite de R devient :

$$
R \sim \pi^{2}\left(\frac{b}{V}\right)^{2} \frac{1}{k^{4}}
$$

Dans le cas des plusieurs films déposés sur un substrat, il faut tenir compte que le faisceau est réfléchi dans chaque couche. Les coefficients de Fresnel vont devoir tenir compte des différents milieux. Dans cette géométrie le coefficient de réflexion combiné- $r_{n-2, n}$-est lié au substrat (n) ainsi qu' aux couches n-1 et n-2 et il est donc donné par :

$$
r_{n-2, n}=\frac{r_{n-2, n-1}+r_{n-1, n} e^{2 i k_{n-1} d_{n-1}}}{1+r_{n-2, n-1} r_{n-1, n} e^{2 i k_{n-1} d_{n-1}}}
$$

où $\mathrm{r}_{i, j}$ est le coefficient de réflexion de Fresnel pour l'interface entre milieu i et $\mathrm{j}$, $\mathrm{d}$ est l'épaisseur de la couche et $\mathrm{k}_{\mathrm{i}}$ est le composant $\mathrm{z}$ du vecteur d'onde. Ce processus récursif peut s'étendre jusqu'à la surface et la réflectivité est donc obtenue. Cependant entre deux milieux semi-infinis l'interface ne va pas être parfaitement plane, on aura une certaine rugosité. On peut considérer ce phénomène en décrivant le profil de l'interface par la convolution de l'interface avec une fonction Gaussienne [28] :

$$
R_{R}(k)=R_{F}\left(k_{z}\right) \exp \left(-4 k^{2} \sigma^{2}\right)
$$

où $\sigma$ correspond à la rugosité de l'interface, $\mathrm{z}$ la distance de l'interface et $\mathrm{R}_{\mathrm{F}}$ la réflectivité en absence d'un gradient de composition à l'interface. Dans cette géométrie le coefficient de réflexion lié aux couches $n$ et n-1 est donné par:

$$
r_{n, n+1}=\left(\frac{k_{n}-k_{n+1}}{k_{n}+k_{n+1}}\right) \exp \left(-2 \sigma_{n+1}^{2} k_{n} k_{n+1}\right) .
$$

En mesurant la réflectivité on peut donc obtenir des informations sur la rugosité des surfaces et interfaces. Le profil interfacial d'une couche mince entre deux lieux semi-infinis peut être déterminé en considérant la relation :

$$
\rho(z)=\sum_{i=1}^{n} \frac{\rho_{i}-\rho_{i+1}}{2}\left(1+\operatorname{erf}\left(\frac{\mathbf{z}-\mathbf{z}_{i}}{\sqrt{2} \sigma_{i}}\right)\right)
$$

où $\rho_{\mathrm{i}}, \sigma_{\mathrm{i}}$ et $\mathrm{z}_{\mathrm{i}}$ sont respectivement la densité de la longueur de diffusion, la rugosité et la distance de la surface pour la couche i. La fonction erf est une fonction erreur. Enfin on peut relier cette rugosité à l'épaisseur interfaciale définie pour un profil tangentiel hyperbolique [15] :

$$
w=\sqrt{\frac{\pi}{2}} \sigma .
$$

Les profils de réflectivités des neutrons ont été mesurés sur de nombreux réflectomètres : D17 à l'Institut Laue Langevin (Grenoble, France), CRISP au Rutherford Appleton Laboratory (Didcot, UK), V6 au Hein-Meitner Institute (Berlin, Germany), AMOR au Paul Sherrer Institute (Villigen, Suisse), et sur le réflectomètre EROS au Laboratoire Léon Brillouin (Saclay, France). Les résolutions utilisées ont varié entre 3 et $5 \%$. Ensuite les profils obtenus sur les bicouches ont été traités en utilisant un modèle composé d'un substrat de silicium/oxyde de silice/polymère deutéré/polymère hydrogéné/air, avec une rugosité Gaussienne à la surface et à l'interface polymère/polymère. Dans cette configuration, comme le film hydrogéné a une très basse densité de longueur de diffusion, il n'y a pas de contraste entre l'air et la surface supérieure des échantillons. Donc les mesures de réflectivité ne sont pas sensibles à la valeur de la rugosité de la surface. Pour les copolymères deutérés, la valeur de $\rho$ est autour de $3 \times 10^{-6} \AA^{-2}$, 


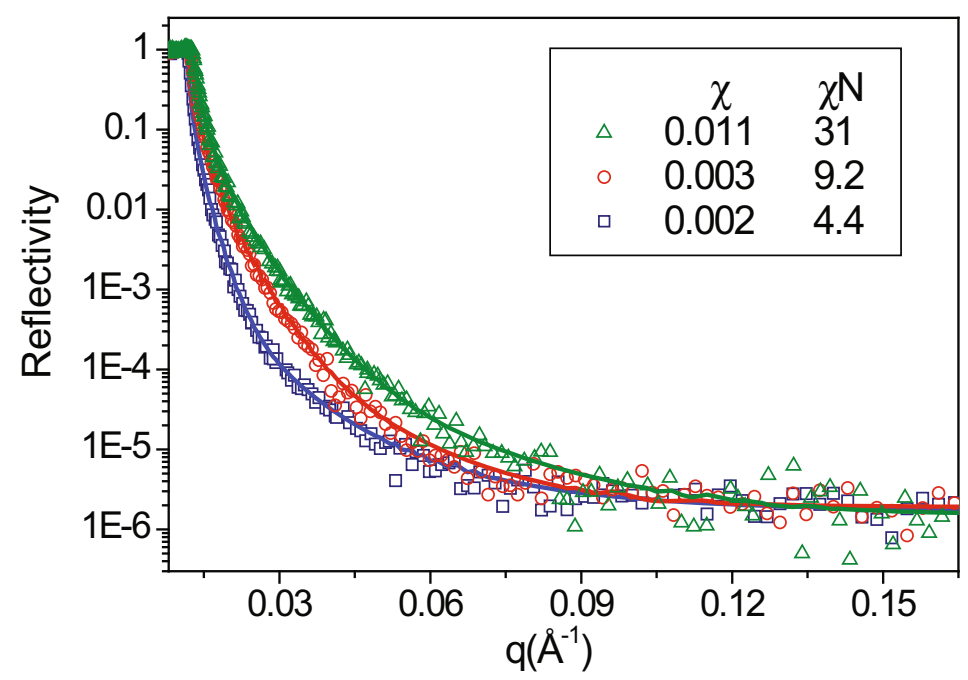

Figure 1. Profils de réflectivité pour des paires de copolymères avec différents paramètres d'interaction $\chi$, et un degré de polymérisation $\mathrm{N} \sim 2500$. Les épaisseurs des films sont comprises entre 2600 et $5000 \AA$, dans ce cas les franges de Kiessig sont indiscernables.

alors que pour le mélange hydrogéné, il est compris entre $2 \times 10^{-7} \AA^{-2}$ et $-3 \times 10^{-7}$, $\AA^{-2}$. Afin d'étudier comment l'épaisseur de l'interface atteint son équilibre pour chaque degré d'immiscibilité, les échantillons sont chauffés durant différents temps variant de 5 min à 5 jours, à une température de $83^{\circ} \mathrm{C}$, bien supérieure à la transition vitreuse.

\section{DÉPENDANCE DE L'INTERFACE PAR RAPPORT À LA DISTANCE AU POINT CRITIQUE}

Pour examiner la dépendance de la largeur de l'interface par rapport à la valeur de son paramètre d'immiscibilité et au point critique de miscibilité, des couples de copolymères hydrogénés et partiellement deutérés ont été étudiés par réflectométrie des neutrons. Dans ces expériences, la masse moléculaire a été fixée $(150,000 \mathrm{~g} / \mathrm{mol})$ et différentes conditions de miscibilité ont été examinées. Les épaisseurs des couches sont comprises entre $3000 \AA$ At $6000 \AA$, afin d'éviter des effets de confinement. La figure 1 montre un exemple de profils de réflectivité mesuré pour une bicouche $(\mathrm{D} / \mathrm{H})$ : on peut observer qu'après la fin du plateau critique $\mathrm{q}_{c} \sim 0.0125 \AA^{-1}$, l'intensité des profils diminue aussi rapidement que le degré d'immiscibilité décroit. Cette diminution correspond à une interface plus rugueuse. Ce phénomène est d'autant plus marqué quand le paramètre d'interaction passe de 0.0033 à 0.0018 .

La figure 2 montre la dépendance de l'épaisseur de l'interface à l'équilibre pour des bicouches de polymères en fonction du paramètre de miscibilité $\chi \mathrm{N}$. On observe la divergence de l'interface au point critique: dans la région $\chi \mathrm{N}<8$, la croissance de l'épaisseur de l'interface est plus forte au fur et mesure que le paramètre d'immiscibilité devient plus important, et vers $\chi \mathrm{N}=2$ l'interface diverge [31]. La théorie des champs moyens présentée dans la section précédente a été utilisée pour l'analyse des données. Le modèle utilisé décrit l'interface dans le cas de la «Strong-Segregation-Limit» (SSL), où les deux polymères sont fortement immiscibles ( $\chi \mathrm{N}$ élevé). Quand l'interface devient proche du point critique $\chi \mathrm{N}=2$ l'interface diverge et l'utilisation de la théorie des champs moyens est à reconsidérer: pour les faibles $\chi \mathrm{N}$ la théorie WSL est donc utilisée. Dans le but de comparer les résultats expérimentaux aux prédictions théoriques, la contribution des ondes capillaires doit être prise en compte. Le produit 


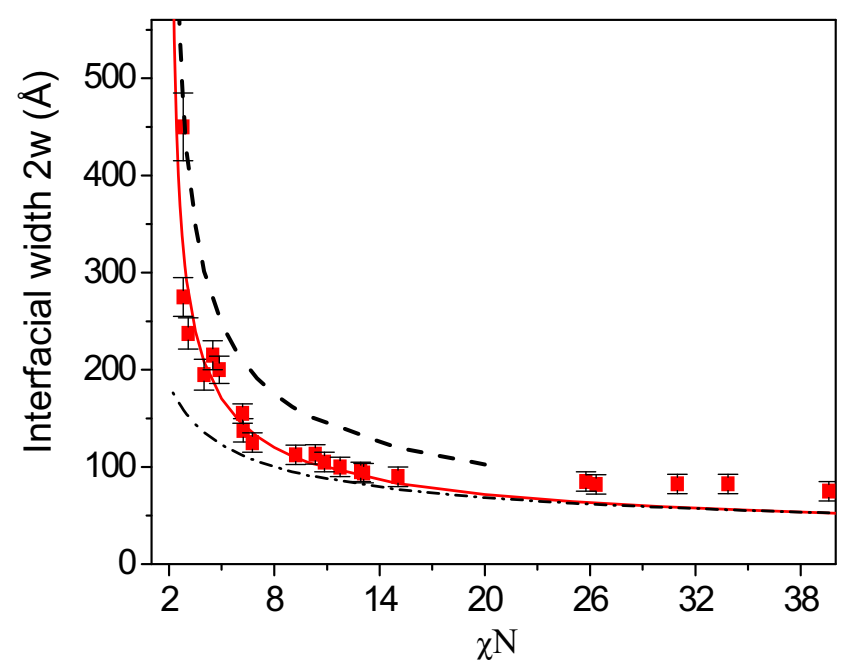

Figure 2. Epaisseur de l'interface en fonction du degré d'immiscibilité. Le paramètre d'interaction varie lorsque le degré de polymérisation est fixé à 2 500. Les épaisseurs de ces systèmes sont comprises entre $3000 \AA$ et $6000 \AA$. La ligne en traits discontinus correspond à l'utilisation de la WSL, et la courbe en trait et point alternés à la SSL. Les carrés rouges correspondent à l'interface à l'équilibre de bicouche de polyoléfine pour différents degrés d'immiscibilité.

$\pi \mathrm{w}_{I}$ a été utilisé comme borne supérieure pour les vecteurs d'onde avec de petites longueurs d'onde [32]. Donc la contribution des ondes capillaires à l'interface est donnée par :

$$
\sigma_{\varsigma}^{2}=\frac{k_{B} T}{2 \pi \gamma} \ln \frac{\lambda_{c o h}}{\pi w_{I}}
$$

où $k_{B}$ est la constante de Boltzmann, T est la température, $\lambda_{c o h}$ est la longueur de cohérence latérale du faisceau de neutrons et $\gamma$ est la tension interfaciale. Dans la SSL $w_{I}$ et $\gamma$ sont déterminés en utilisant respectivement les équations 5 et 6 , alors que pour la WSL les expressions 10 et 11 ont été utilisées. Dans les calculs, la valeur de $a$ est de $6 \AA$ et le volume moyen par monomère est $75 \AA^{3}$. Une estimation théorique de l'interface totale a été ensuite obtenue en utilisant la valeur estimée de $\sigma_{\varsigma}^{2}$ avec l'équation 29 dans l'équation 15.

Des exemples d'épaisseurs d'interfaces calculées à partir de ces deux limites sont repris sur la figure 2. La ligne en trait discontinu correspond à l'utilisation de la WSL, et la courbe en traits et points alternés à la SSL, l'épaisseur de l'interface totale tient compte de l'interface intrinsèque et des fluctuations des ondes capillaires. Il apparait que pour les haut $\chi \mathrm{N}$, la SSL décrit le comportement des interfaces. Mais pour des degrés d'immiscibilité proches du point critique l'interface suit la description WSL. Il existe une zone intermédiaire pour le degré d'immiscibilité, où ni la SSL et la WSL ne représentent l'interface.

\section{SYSTÈMES CONFINÉS}

Dans le cas où la dimension d'un système est réduite, les molécules de polymères manifestent souvent des propriétés déviantes par rapport au matériau en «volume». Dans les films minces de polymères, avec une épaisseur inférieure au rayon de giration de la molécule, des différences de propriétés structurales et dynamiques ont été observées expérimentalement et par simulation [33-37]. Celles-ci incluent par exemple une variation de la température vitreuse avec la décroissance de l'épaisseur du film, une mobilité différente des chaînes avec une plus grande compressibilité dans les films les plus minces. De 


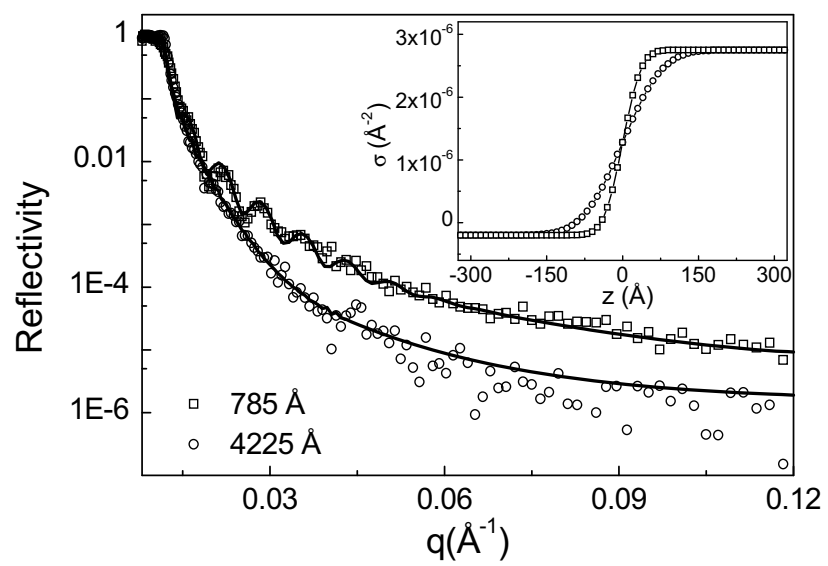

Figure 3. Courbes de réflectivité de neutrons pour des bicouches de polyoléfines. La couche supérieure est hydrogénée, le copolymère ratio est de $86 \%$, et l'épaisseur autour de $4000 \AA$ A pour les deux échantillons. La couche inférieure est partiellement deutérée, le copolymère ratio est $70 \%$, et les épaisseurs sont $785 \AA$ (carrés) et 4225 Å (cercles). Les régressions utilisant des modèles à trois couches sont montrées par les lignes. La figure insérée représente les profils de densités de longueur de diffusion pour les deux courbes correspondant aux régressions.

plus il a été montré que l'effet dû aux confinements et aux interactions moléculaires dans la géométrie de film mince est souvent responsable de nouveaux phénomènes physiques. Pour citer un exemple, les interactions intermoléculaires à longue portée peuvent affecter la stabilité ou l'instabilité de films minces en dessous d'une certaine épaisseur [38], allant jusque dans certains cas à du démouillage spinodal [39] et à la formation de motifs structurels caractérisées par une longueur caractéristique bien définie [40]. Le confinement géométrique a également un effet important sur les interfaces entre deux polymères immiscibles. Des expériences effectuées par différents groupes ont montré que la largeur de l'interface entre deux films minces dépend de l'épaisseur des films. Pour les films très minces le spectre des ondes capillaires est modifié par la contrainte du confinement, et l'épaisseur de l'interface est plus petit. Cependant, il y a une controverse dans la littérature concernant la relative importance des forces à courte et longue portée dans la modification des fluctuations de l'interface. Les simulations numériques de la dépendance de la taille du profil de l'interface entre deux milieux de polymères confinés suggèrent que ces deux forces doivent être en principes prises en compte [41]. Ces études ont montré que les longueurs d'ondes capillaires les plus longues sont déterminées par une longueur de corrélation parallèle qui incorpore deux contributions: la première est à courte portée, et provient de la distorsion du profil proche de la paroi, la seconde est à longue portée, et est due aux interactions de Van der Waals. Cependant l'influence mutuelle entre les forces à courte et longue portée à l'interface n'est pas bien comprise. Or leur rôle est déterminant dans la largeur de l'interface de films minces. Le mécanisme par lequel l'effet de taille finie influence l'épaisseur de l'interface entre deux polymères a été étudié en réalisant des expériences de réflectivité de neutrons sur des bicouches de polyoléfines hydrogénées et partiellement deutérés. L'épaisseur des couches supérieures est comprise entre 2600 $\AA$ et $5000 \AA$, celle des couches inférieures varie dans une gamme de 700 à $9000 \AA$. La figure 3 est un exemple de courbes de réflectivités obtenues pour un couple de polymères avec un degré d'immiscibilité $\chi \mathrm{N}=6.2$, et $\mathrm{N} \sim 2000$, pour deux épaisseurs différentes de la couche inférieure: $785 \AA$ (symbole carré) et $4225 \AA$ (symbole cercle). Dans le premier profil les oscillations caractéristiques de l'épaisseur de la couche inférieure sont clairement visibles. Comme l'épaisseur de la couche inférieure augmente, les oscillations disparaissent et l'intensité de la réflectivité, pour des valeurs de q plus grande que $\mathrm{q}_{c}$, diminue rapidement, indiquant une interface plus large pour une couche plus grande. 


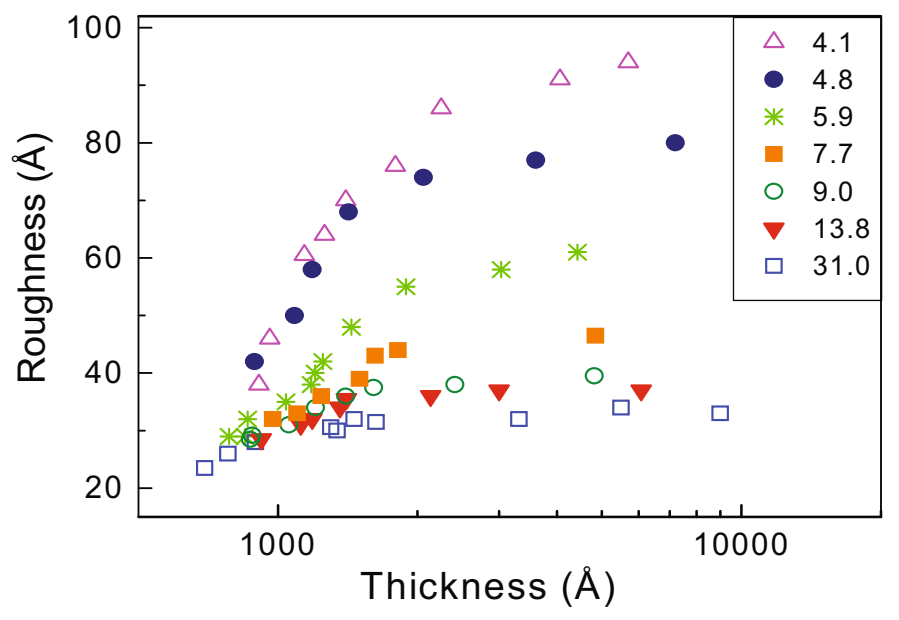

Figure 4. Rugosités interfaciales en fonction de l'épaisseur de la couche inférieure et du degré d'immiscibilité pour tous les systèmes mesurés par réflectivité de neutrons. La légende montre les différentes valeurs de $\chi \mathrm{N}$. Les régressions utilisent le modèle des ondes capillaires décrit dans le texte pour les deux cas extrêmes.

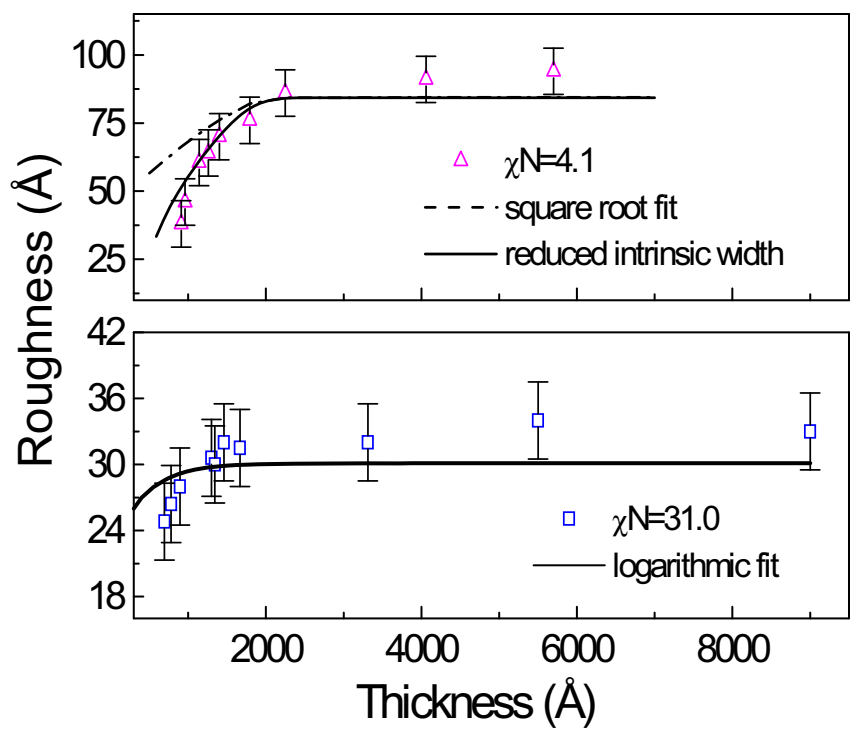

Figure 5. Rugosités interfaciales en fonction de l'épaisseur de la couche inférieure pour $\chi \mathrm{N}=31$ (en bas) et $\chi \mathrm{N}=4.1$ (en haut). Les régressions des points sont aussi montrées dans la figure pour le cas des forces de longue portée (en bas) et de courte portée (en haut). Voir le texte pour plus de détails.

Les profils de longueur de diffusion extraits par les régressions sont montrés dans la figure insérée. A partir des régressions des profils de réflectivité, les rugosités des interfaces sont représentées sur la figure 4 en fonction de l'épaisseur de la couche inférieure et du degré d'immiscibilité. Pour tous les différents $\chi \mathrm{N}$, la rugosité augmente avec l'épaisseur de la couche supérieure à une valeur limite, stable dans le temps. En comparant les systèmes avec les différents $\chi \mathrm{N}$, la rugosité de l'interface augmente plus rapidement pour les polymères faiblement immiscibles avec l'augmentation de la couche inférieure.

En outre la valeur limite de l'interface est supérieure avec les polymères faiblement immiscibles. De plus, quand le degré d'immiscibilité décroît, l'interface devient plus large, et la dépendance avec 


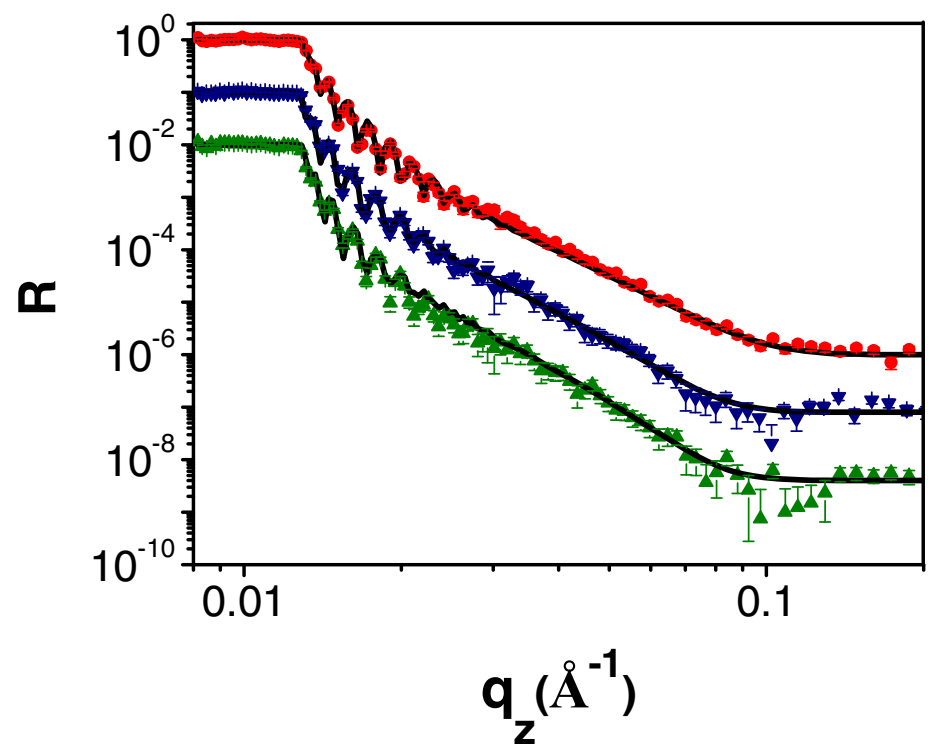

Figure 6. Exemples de profils de courbes de réflectivité pour une bicouche de copolymère avec comme paramètre $\chi \mathrm{N}=3.7$. Les épaisseurs sont autour de $400 \AA$ A, trois différents temps de chauffages sont représentés: 0 (cercle), $25 \mathrm{~min}$ (triangle inversé), et $265 \mathrm{~min}$ (triangle). Les courbes noires sont leurs régressions. Les différentes courbes sont translatées d'un facteur 10 sur l'axe des y pour plus de visibilité.

l'épaisseur de la couche inférieure devient plus forte. La figure 5 montre plus en détail la rugosité de l'interface entre les deux polymères en fonction de l'épaisseur de la couche inferieure pour le cas immiscible $(\chi \mathrm{N}=31)$ et le cas moins immiscible $(\chi \mathrm{N}=4.1)$.

Pour le système avec $\chi \mathrm{N}=31$, une régression des points donne une dépendance logarithmique de la largeur de l'interface en fonction de l'épaisseur du film comme prédit pour les forces à longue portée tandis que pour $\chi \mathrm{N}=4.1$ il y a une dépendance de la rugosité de l'interface en fonction de la racine carrée de l'épaisseur (avec éventuellement une interface intrinsèque réduite) comme prédit pour le cas des forces à courte portée [26, 42, 43].

\section{FORMATION DE L'INTERFACE}

Comme mentionné précédemment, les chaînes de polymère ont une dynamique lente, il est donc possible de comprendre comment l'interface atteint son état d'équilibre. La formation de l'interface intrinsèque est très rapide - elle est reliée directement à la mobilité des chaines - tandis que la croissance des ondes capillaires, plus lente, peut être suivie en détail. Pour étudier ce processus, des expériences de réflectométrie des neutrons ont été effectuées pour mesurer l'épaisseur de l'interface polymère/polymère en fonction du temps de chauffage de différents systèmes de polyoléfine avec divers degrés de miscibilité. La figure 6 montre un exemple de courbe de réflectivité pour les systèmes avec un $\chi \mathrm{N}=3.7$ et différents temps de chauffage: trois temps de chauffages sont représentés: 0 min (cercle), 25 min (triangle inversé), et 265 min (triangle). Les courbes sont translatées d'un facteur 10 sur l'axe des y pour plus de visibilité. Les franges d'interférences sont reliées à l'épaisseur de la couche supérieure. Une décroissance de l'intensité de la réflexion est observée. Pour le $\chi \mathrm{N}=18$, toutes les courbes à différents temps de chauffage se superposent. Ces résultats montrent déjà que pour le système avec le plus faible degré d'immiscibilité, la contribution des fluctuations thermiques dans l'épaisseur totale de l'interface est plus importante, et qu'il faut plus de temps pour atteindre l'équilibre. Alors que pour le système 


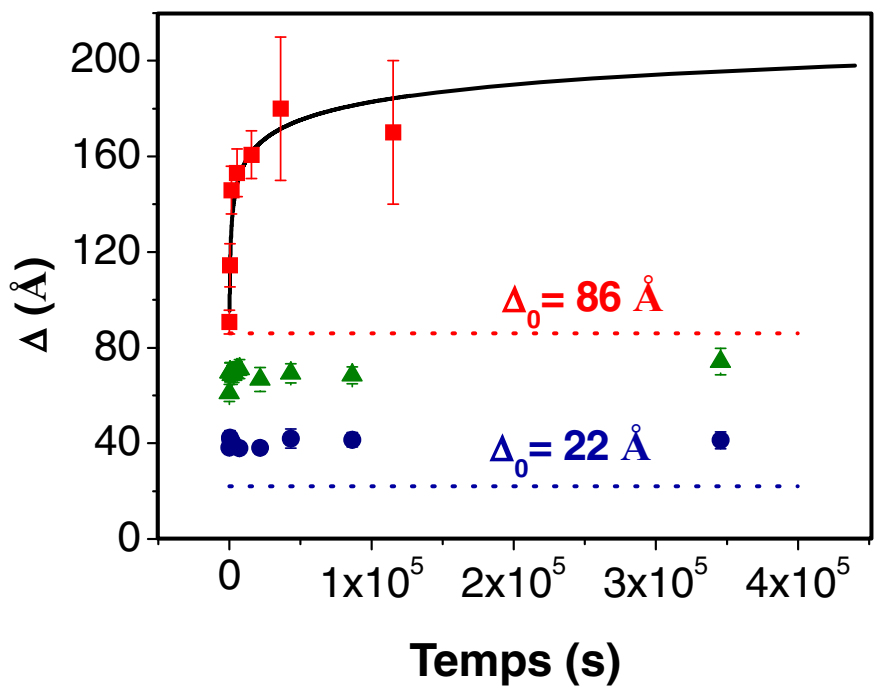

Figure 7. Rugosité de l'interface en fonction du temps de chauffages. Les systèmes sont des bicouches de polyoléfines avec 3 différents degrés d'immiscibilité: 3.7 (carré), 6.8 (triangle), 18 (cercle). Les lignes en pointillé sont les prédictions de la rugosité intrinsèque prédit par la «Self-Consistent-Theory » dans la «Strong-SegregationLimit » pour $\chi \mathrm{N}=18$, et dans la «Weak-Segregation-Limit» pour $\chi \mathrm{N}=3.7$. La ligne pleine représente un fit tenant compte de la contribution des ondes capillaires dans la formation de l'interface.

avec le plus haut degré d'immiscibilité, l'équilibre de l'interface est atteint plus rapidement. Les lignes pleines de la figure 6 représentent les régressions obtenues par la méthode des moindres carrés pour un modèle tri-couches avec une rugosité gaussienne pour la surface et l'interface polymère/polymère. En résumé, lors de ces expériences 3 degrés d'immiscibilités ont été employés: $\chi \mathrm{N}=3.7$ (carré), 6.8 (triangle), et 18 (cercle). La rugosité de l'interface polymère/polymère est représentée en fonction du temps de chauffage à $83^{\circ} \mathrm{C}$ sur la figure 7 . Les lignes en pointillé sont les prédictions de la rugosité intrinsèque prédit dans la SSL pour $\chi \mathrm{N}=18$, et dans la WSL pour $\chi \mathrm{N}=3$.7. La ligne pleine représente une régression tenant compte de la contribution des ondes capillaires dans la formation de l'interface. On observe que pour le plus bas $\chi \mathrm{N}$, la rugosité augmente avec le temps jusqu'à une valeur d'équilibre. En comparant les différents systèmes, pour celui avec le plus haut degré d'immiscibilité, l'interface est déjà à sa valeur d'équilibre dès le départ. La rugosité intrinsèque calculée dans le cas de la SSL est de 22 $\AA$. La partie de l'interface due aux fluctuations thermique est de $30 \AA$, pour une tension interfaciale de $8 \times 10^{-4} \mathrm{~J}_{\mathrm{m}^{-2}}$. Dans ce système, l'amplitude des ondes capillaires atteint son équilibre pour des temps courts.

Dans le cas du degré d'immiscibilité de 6.8, le système atteint assez rapidement un équilibre de 68 Å. Ce cas ne peut être décrit ni par la SSL ni par la WSL, il se situe donc dans un régime intermédiaire. Pour le plus faible degré d'immiscibilité (3.7), la WSL a été utilisée pour suivre le comportement de l'interface. La rugosité initiale est de $91 \AA$, alors que la rugosité intrinsèque calculée est de $86 \AA$. L'estimation du terme de l'onde capillaire donne $30 \AA$, une valeur similaire à celle du plus fort degré d'immiscibilité. Ensuite il y a une croissance de la rugosité qui correspond à la croissance du terme de l'onde capillaire. La rugosité intrinsèque déduite par régression est de $86 \pm 3 \AA$, la tension superficielle de $2.0 \pm 0.5 \times 10^{-5} \mathrm{Jm}^{-2}$, et une viscosité de $3.0 \pm 0.5 \times 10^{5} \mathrm{~Pa}$ s. La rugosité intrinsèque déduite est similaire à celle calculés par la WSL, la tension superficielle calculée est de $5 \times 10^{-5} \mathrm{Jm}^{-2}$, ce qui est dans le même ordre de grandeur [44]. 


\section{CONCLUSIONS}

Le chapitre a montré l'importance de la réflectivité des neutrons pour clarifier la structure de l'interface de couches minces de polymères. On observe une divergence de l'interface proche du point critique : pour les haut $\chi \mathrm{N}$, la «Strong Segregation Limit» décrit le comportement des interfaces, mais pour des degrés d'immiscibilité proches du point critique l'interface suit la description WSL. Il existe une zone intermédiaire pour le degré d'immiscibilité, où ni la SSL et la WSL ne représentent l'interface. L'étude du confinement sur les ondes capillaires aux interfaces a également été présenté. Les effets des forces à longue portée et de courte portée sur l'épaisseur de l'interface ont été mis en évidence. Enfin la formation d'une interface polymère/polymère a été étudiée. Il a été vu que dans la formation de l'interface, il y a une partie qui est due à l'interface intrinsèque entre les deux polymères, et l'une autre-aux fluctuations thermiques, les ondes capillaires : pour de plus larges interfaces il y a une lente augmentation de l'interface directement relié à la croissance des ondes capillaires.

\section{Remerciements}

À N. Brouette (Université Libre de Bruxelles), C. Carelli (Surrey University), RAL Jones et RN Young (University of Sheffield). Remerciements aussi à R. Cubitt et G. Fragneto (ILL), R. Krastev (HMI), T. Gutberlet (PSI), F. Cousin (LLB), R. Dalgliesh (RAL) pour les expériences de neutrons.

\section{Références}

[1] K. Binder, Advances in Polymer Science, 1999, 138, 1

[2] R. A. L. Jones and R. W. Richards, Polymers at Surfaces and Interfaces, Cambridge University Press, 1999

[3] M. Sferrazza, et al., Physical Review Letters, 1998, 81, 5173

[4] E. Helfand, Y. Tagami, J. Chem. Phys., 56, 3592 (1971)

[5] K. Shull et al., Macromolecules 26, 3929 (1993)

[6] A. N. Semenov, Macromolecules 27, 2732 (1994)

[7] H. Tang, K. Freed, J. Chem. Phys., 94, 6307 (1991)

[8] I. C. Sanchez, Physics of Polymer Surfaces and Interfaces, Butterworth-Heinemann, Boston (1992)

[9] J. W. Cahn, J. E. Hilliard, J. Chem Phys., 28,258 (1958)

[10] F. Schmid, J. Phys. Condens. Matter, 10, 8105 (1998)

[11] H. Tang, K. Freed, J. Chem. Phys., 94, 6307 (1991)

[12] J. W. Cahn, J. E. Hilliard, J. Chem Phys., 28,258 (1958)

[13] J. S. Rowlinson and B. Widom, Molecular theory of capillarity, Clarendon Press, 1982.

[14] F. P. Buff et al., Phys. Rev. Lett., 15,621 (1965

[15] M. Sferrazza, et al., Physical Review Letters, 1997, 78, 3693

[16] M. Sferrazza et al., J. Cond. Mat. C 13, 10269 (2001)

[17] M. Sferrazza et al., Phys. Mag. Lett., 80, 561 (2000)

[18] J. Israelachvili, Intermolecular and surface forces, Academic Press, London (1985)

[19] A. O. Parry, R Evans, Phys. Rev. lett., 64, 439 (1990)

[20] A. O. Parry, R Evans, Physica A, 181, 250 (1992)

[21] R. Evans, A. O. Parry, J. Phys. Cond. Matt., 1, 7207 (1989)

[22] K. Binder et al., Phys. rev. E, 51, 2823 (1995)

[23] K. Binder et al., J. Stat. Phys. 95, 1045 (1999)

[24] K. Binder et al., Adv. Polym. Sci., 112, 181 (1994)

[25] T. Kerle, J. Klein, and K. Binder, Physical Review Letters, 1996, 77, 1318.

[26] C. Carelli et al., Europhysics Letter, 71, 763-769 (2005)

[27] W. W. Graessley, et al., Macromolecules 27, 3896 (1994) 
[28] T. P. Russell, Mater. Sci. Progr., 5, 171, (1990)

[29] J. Penfold, R. K. Thomas, J. Phys. Cond. Matt., 2, 1369 (1990)

[30] G. Gragneto-Cusani, J. of Phys.: Cond. Matt. 13, 4973 (2001)

[31] C. Carelli et al., Phys. Rev. E, 72, 031807 (2005)

[32] A. N. Semenov, Macromolecules, 26, 6617 (1993)

[33] J. Keddie et al., Europhys. Lett., 27, 59 (1994)

[34] J. A. Forrest, R. A. L. Jones, Adv. Coll. Int. Sci., 94, 167 (2001)

[35] H. R. Brown, T. P. Russell, Macromolecules, 28, 6808 (1996)

[36] K. Binder et al., Ann. Rev. Mat. Sci., 26, 107 (1996)

[37] F. Varnik et al., Eur. Phys. J. E, 8, 175 (2002)

[38] G. Reiter, Phys. Rev. Lett., 68, 1084 (1992)

[39] M. Sferrazza et al., Phys. Rev. Let. 81, 5173-5176 (1998)

[40] A. M. Higgins, RAL Jones, Nature, 404, 476 (2000)

[41] A. Werner et al., J. Chem. Phys., 110, 1221 (1999)

[42] C. Carelli, R. N. Young, R. A. L. Jones and M. Sferrazza, Europhy Let. 75, 274 (2006)

[43] M. Sferrazza and C. Carelli, J. Condensed Matter Physics 19, 033101 (2007)

[44] W. Béziel, G. Fragneto, F. Cousin and M. Sferrazza, Phys. Rev. E 78, 022801 (2008) 